\title{
All-Path Decoding Algorithm for Segmental based Speech Recognition
}

\author{
Yun TANG, Wen-Ju LIU, and Bo XU \\ National Laboratory of Pattern Recognition, \\ Institute of Automation, Chinese Academy of Sciences. \\ hottang@gmail.com, lwj@nlpr.ia.ac.cn
}

\begin{abstract}
In conventional speech processing, researchers adopt a dividable assumption, that the speech utterance can be divided into nonoverlapping feature sequences and each segment represents an acoustic event or a label. And the probability of a label sequence on an utterance approximates to the probability of the best utterance segmentation for this label sequence. But in the real case, feature sequences of acoustic events may be overlapped partially, especially for the neighboring phonemes within a syllable. And the best segmentation approximation even reinforces the distortion by the dividable assumption. In this paper, we propose an all-path decoding algorithm, which can fuse the information obtained by different segmentations (or paths) without paying obvious computation load, so the weakness of the dividable assumption could be alleviated. Our experiments show, the new decoding algorithm can improve the system performance effectively in tasks with heavy insertion and deletion errors.
\end{abstract}

\section{Introduction}

In the framework of statistics, the mapping between the label sequence $l_{1}^{N}=$ $\left\{l_{1}, l_{2}, \cdots, l_{N}\right\}$ and one speech observation sequence $x_{1}^{T}$ is determined by the rule of maximum a posterior probability (MAP).

$$
\begin{aligned}
l_{1}^{N} & =\arg \max _{l_{1}^{N}, N} P\left(l_{1}^{N} \mid x_{1}^{T}\right), \\
& =\arg \max _{l_{1}^{N}, N} P\left(x_{1}^{T} \mid l_{1}^{N}\right) P\left(l_{1}^{N}\right),
\end{aligned}
$$

where, $P\left(x_{1}^{T} \mid l_{1}^{N}\right)$ is the acoustic model score and $P\left(l_{1}^{N}\right)$ is the language model score for the label sequence $l_{1}^{N}$. Each label in $l_{1}^{N}$ represents an acoustic event, such as phonme, and is exhibited by a corresponding segment in $x_{1}^{T}$. The corresponding segments for neighboring labels may be disjunct, or adjacent, or even overlapped. For example, the corresponding segments for label sequence $\{a, b, c, d, e\}$ are shown in fig.1(I), where "sil" means that there exists silence between labels. Shadowed parts in fig.1(I) indicate that these regions in time axis are shared by more than one acoustic event. The corresponding observation segments for $a$ and $b$ are disjunct; the corresponding observation segments for $b$ 
and $c$ are overlapped partially. For the convenience of modeling and decoding, a dividable assumption is adopted in the state-of-the-art of acoustic model, that is, the corresponding segment for each label is dividable in time axis and no overlapping region exists, as fig.1(II) shows. By this assumption, the influence of each acoustic event is limited in its corresponding observation segment, such as the context independent acoustic model; or it may also affect neighboring observation segments, such as the context dependent acoustic model. Then, the likelihood probability of a label sequence matching an observation sequence could be dissolved into likelihood scores of labels on their corresponding segments.

(I)
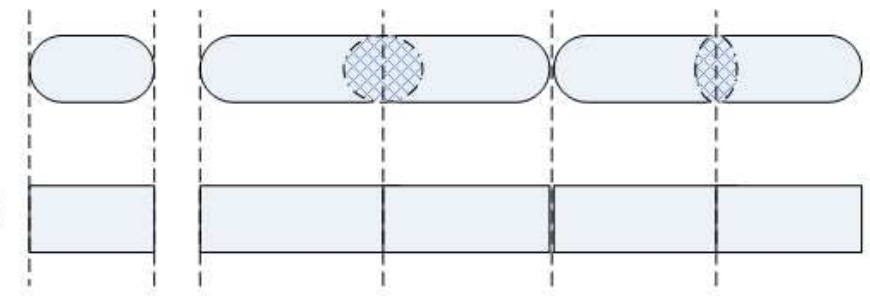

(sil)

a (sil)

b

C

d

e (sil)

Fig. 1. Dividable assumption in speech processing. (I) The overlapping between acoustic events; (II) the segmentation after the dividable assumption is applied.

$$
\begin{array}{r}
P\left(x_{1}^{T} \mid l_{1}^{N}\right)=\sum_{\substack{B_{0}^{N} \in \Lambda_{T, N} \\
B_{i-1}<B_{i}, B_{0}=0, B_{N}=T,}}^{N} P\left(x_{B_{(i-1)}+1}^{B_{i}} \mid l_{i}\right),
\end{array}
$$

where $\Lambda_{T, N}$ is the segmentation set for an observation sequence with $T$ frames and $N$ labels, $\left(B_{i-1}+1\right)$ and $B_{i}$ are the boundary frames of the $i$-th label. As $N$ and $T$ increasing, the number of possible segmentations is increased exponentially. So it is infeasible to count all segmentation cases to find the most likely label sequence for a given observation sequence. An usual way is to choose the label sequence with the highest probability segmentation as an approximate result of Equ.(2)

$$
P\left(x_{1}^{T} \mid l_{1}^{N}\right)=\max _{B_{0}^{N} \in \Lambda_{T, N}} \prod_{i=1}^{N} P\left(x_{B_{i-1}+1}^{B_{i}} \mid l_{i}\right) .
$$

As mentioned above, the boundary between neighboring labels may be overlapped or even not exist for certain label pairs, such as the virtual syllable initial and syllable final pairs for non-initial syllables ${ }^{1}$ in Mandarin[1]. Hence, the divid-

\footnotetext{
${ }^{1}$ The syllables only consist of syllable final and tone in Chinese characters, such as digit "2".
} 
able assumption does not represent the real situation of human utterances. The best path approximation in Equ.(3) ignores other possible segmentations and reinforces the distortion introduced by the dividable assumption. In this paper, we propose an all-path decoding algorithm, which can fuse the information obtained by different segmentations or paths without paying obvious computation load, so the weakness of the dividable assumption could be alleviated. In order to distinguish the all-path decoding from the conventional method, the decoding based on Equ.(3) is called the best path decoding in this paper. Our experiments show, the best path approximation has no obvious influence on common tasks, while the all-path decoding can effectively improve the system performance in tasks with heavy reduction and deletion errors.

The rest of this paper is organized as follows. A brief introduction of Stochastic Segment Model(SSM) [2], the acoustic model adopted in the prototype system, is given in Section 2. Then in Section 3, the all-path decoding algorithm is presented in detail. Section 4 shows the experimental results and analysis on the mandarin digit string and mandarin large vocabulary continue speech recognition (LVCSR) tasks respectively. Conclusions are drawn in the last section.

\section{Stochastic segment model}

Segment model (SM)[3] is a family of methods that adopt segmental distribution rather than frame-based features to represent the underlying trajectory of the observation sequence. The biggest difference between HMM and SM is that HMM models and decodes the utterance in a frame-based way while SM is in a segment-based way. A segment in SM can present a phoneme, a syllable or a word etc. The decoding algorithm of SM is directly based on Equ.(3).

SSM is one kind of SM. Each segment model in SSM has a fixed length region sequence $r_{1}^{L}$, which is used to represent the variable length observation sequence $x_{\tau_{1}}^{\tau_{2}}$. A re-sample function is needed to uniform $x_{\tau_{1}}^{\tau_{2}}$ to an $L$ length sequence $y_{1}^{L}$ so it can be measured by SSM.

$$
y_{i}=x_{\left\lfloor\frac{i}{L}\left(\tau_{2}-\tau_{1}\right)+\tau_{1}\right\rfloor}, 0<i \leq L,
$$

where $\lfloor z\rfloor$ is the maximum integer no larger than $z$.

The re-sampled frame is measured by region, which is similar to the conception of the state in HMM. The $\log$-likelihood of a segment $x_{\tau_{1}}^{\tau_{2}}$ given model $l_{\alpha}$ is the production of region scores:

$$
\ln \left[p\left(x_{\tau_{1}}^{\tau_{2}} \mid l_{\alpha}\right)\right]=\sum_{i=1}^{L} \ln \left[p\left(y_{i} \mid l_{\alpha}, r_{i}\right)\right]
$$

where $r_{i}$ is the $\mathrm{i}$-th region model in segment model $l_{\alpha}$. Usually, each region is characterized by a Gaussian mixture model.

From Equ.(3), the decoding process for utterance $x_{1}^{T}$ is as follows:

$$
J^{*}(m)=\max _{\tau, l_{\alpha}}\left\{J^{*}(\tau)+\ln \left[p\left(x_{\tau}^{m} \mid l_{\alpha}\right)\right] \cdot(m-\tau)+C\right\},
$$


where $C$ is the insertion factor, $J^{*}(m)$ is the accumulated score of the best acoustic model sequence at frame $m$ and $J^{*}(0)$ is initialized to 0 . A candidate set and an expanding set are formed at each frame during decoding. The candidate set is a collection of hypothesized paths ending at this point and the expanding set is the collection of acoustic models which succeed the paths in the candidate set. The decoding is performed from 1 to $T$ frame by frame and the decoding result is the label sequence attached to the path with the highest probability in candidate set of $T$.

\section{All-path decoding algorithm}

The all-path decoding algorithm aims to find the probability of a label sequence for an utterance by integrating more information from passible segmentations or paths as Equ.(2) does. In the conventional best path decoding algorithm, a label sequence can reach frame $m$ by multiple paths, which will be merged and only the path with the highest score is survived to the following decoding process. While, in the all-path decoding algorithm, these paths will be fused to form a comprehensive score. Assuming frame $\tau$ is ahead of frame $m$ and we have obtained the probability of label sequence $l_{1}^{n}=\left\{l_{1}, l_{2}, \cdots, l_{n}\right\}$ reaching frame $\tau$ through all paths. We call the probability $P_{A}\left(x_{1}^{\tau} \mid l_{1}^{n}\right)$ as the all-path probability for $l_{1}^{n}$ on $x_{1}^{\tau}$. Then, at condition that the segment $x_{\tau}^{m}$ is labeled as $l_{n+1}$, the probability of label sequence $l_{1}^{n+1}$ on $x_{1}^{m}$ is,

$$
P_{A}\left(x_{1}^{m} \mid \tau, l_{1}^{n+1}\right)=P_{A}\left(x_{1}^{\tau} \mid l_{1}^{n}\right) \cdot P_{A}\left(x_{\tau}^{m} \mid l_{n+1}\right) \cdot P\left(l_{n+1} \mid l_{1}^{n}\right) .
$$

Hence, the all-path probability for labels $l_{1}^{n+1}$ on observation sequence $x_{1}^{m}$ can be obtained by adding all $P_{A}\left(x_{1}^{m} \mid \tau, l_{1}^{n+1}\right) \mathrm{s}$ with different $\tau$ s ahead of $m$, that is,

$$
P_{A}\left(x_{1}^{m} \mid l_{1}^{n+1}\right)=\sum_{\tau=1}^{m-1} P_{A}\left(x_{1}^{m} \mid \tau, l_{1}^{n+1}\right) .
$$

Consequently, the decoding process of SSM is modified according to Equ.(8) as follows,

$$
\begin{array}{r}
J_{A}^{*}\left(m \mid l_{1}^{n+1}\right)=\left\{\ln \sum_{\tau=1}^{m-1} \exp \left[\left(f\left(m \mid \tau, l_{1}^{n+1}\right)\right) / \beta\right]\right\} \cdot \beta, \\
f\left(m \mid \tau, l_{1}^{n+1}\right)=J_{A}^{*}\left(\tau \mid l_{1}^{n}\right)+(m-\tau) \ln p\left(x_{\tau}^{m} \mid l_{n+1}\right)+C,
\end{array}
$$

where $\beta$ is a transfer factor for likelihood probability to probability, $J_{A}^{*}\left(m \mid l_{1}^{n+1}\right)$ is the all-path version of $J^{*}\left(m \mid l_{1}^{n+1}\right)$, and $f\left(m \mid \tau, l_{1}^{n+1}\right)$ is the likelihood version of $P_{A}\left(x_{1}^{m} \mid \tau, l_{1}^{n+1}\right)$. The probability differences among paths will be enhanced when the transfer factor is small, whereas the sub-optimal paths will play more important roles in the all-path probability score. When the value of $\beta$ is close to 0 , the all-path probability will approximate to the maximum likelihood score in 


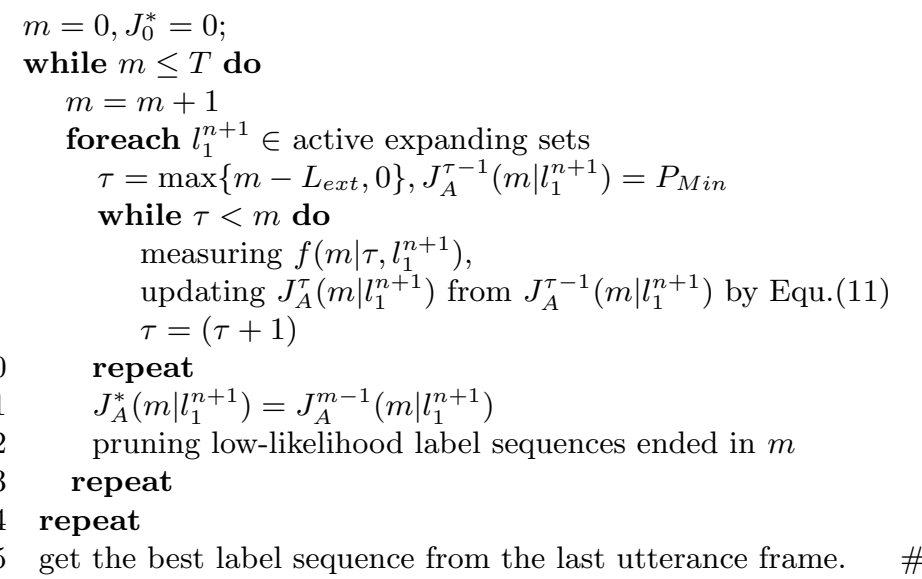

Fig. 2. All-path decoding algorithm.

current paths. So the all-path decoding algorithm will at least achieve a comparable performance with the best path based decoding algorithm. The optimal $\beta$ is chosen by experience in the experiments.

The all-path probability in Equ.(9) is updated in a sequential way. Assuming that the decoder moves to frame $m$ and the current label sequence is $l_{1}^{n+1}$. The initial value of $J_{A}^{*}\left(m \mid l_{1}^{n+1}\right)$ sets to a minimal score $P_{M i n}$; then we count the $\log$ likelihood score $f\left(m \mid \tau, l_{1}^{n+1}\right)$ for each frame $\tau$ before $m$, and these scores update $J_{A}^{*}\left(m \mid l_{1}^{n+1}\right)$ in a sequential way by the difference between $f\left(m \mid \tau, l_{1}^{n+1}\right)$ and $J_{A}^{*}\left(m \mid l_{1}^{n+1}\right)$,

$$
\begin{array}{r}
J_{A}^{\tau}\left(m \mid l_{1}^{n+1}\right)=\ln \left[\exp \left(\left(J_{A}^{\tau-1}\left(m \mid l_{1}^{n+1}\right)-f_{\max }\right) / \beta\right)\right. \\
\left.+\exp \left(\left(f\left(m \mid \tau, l_{1}^{n+1}\right)-f_{\max }\right) / \beta\right)\right] \cdot \beta+f_{\max } \\
f_{\max }=\max \left\{J_{A}^{\tau-1}\left(m \mid l_{1}^{n+1}\right), f\left(m \mid \tau, l_{1}^{n+1}\right)\right\}
\end{array}
$$

where $J_{A}^{\tau-1}\left(m \mid l_{1}^{n+1}\right)$ and $J_{A}^{\tau}\left(m \mid l_{1}^{n+1}\right)$ are all-path probabilities before and after updated by the score $f\left(m \mid \tau, l_{1}^{n+1}\right)$. In this way, the range of $\beta$ could be expanded to a larger scale without consideration of overflowing or underflowing errors. The details of the all-path decoding algorithm is listed in fig.2. $L_{\text {ext }}$ is the allowed maximum segment duration.

Pruning technologies[4][5][6] are applied in the all-path decoding algorithm, since most paths pruned are wrong paths and their probability scores are close to 0 . So it will not make obvious difference whether these wrong paths are considered.

In practice, we assume that two label sequences are same if the last two words are identical. In fact, after pruning, the paths in the expanding set of each frame are originated from a few label sequences and these paths are different from each other mostly by segmentations or the last words. So Equ.(9) can be simplified 
to

$$
J_{A}^{*}\left(m \mid W_{I}, W_{I I}\right)=\left\{\ln \sum_{\tau=1}^{m-1} \exp \left[J_{A}^{*}\left(\tau \mid W_{I}\right)+\ln \left(p\left(x_{\tau}^{m} \mid W_{I I}\right)+C\right) \beta\right]\right\} \cdot \beta,
$$

where $W_{I I}$ is the last word and $W_{I}$ is the word previous $W_{I I}$ in the current label sequence respectively, $J_{A}^{*}\left(\tau \mid W_{I}\right)$ is the maximum all-path probability for the label sequence with the last word $W_{I}$ on frame $\tau$, and $J_{A}^{*}\left(m \mid W_{I}, W_{I I}\right)$ is the all-path score on frame $m$ for the label sequence with $W_{I I}$ and $W_{I}$ as the last two words. Such simplification can seamlessly integrate to a system with a bigram language model. When the trigram model is used, we can trace back to compare the last three words in the current label sequence and a more accurate result can be expected.

\section{Experiments and analysis}

\subsection{Mandarin digit string recognition}

The all-path decoding algorithm was first verified by the mandarin digit string recognition task. Digit string recognition has achieved a satisfied performance in English [7]. However, due to the serious confusion among mandarin digits, the state-of-the-art of mandarin digital string recognition systems does not match that of the English counterpart. Mandarin is a monosyllabic and tonal language, in which a syllable is composed of a syllable initial, syllable final, and tone. Insertion or deletion errors mainly exist in non-syllable initial characters, e.g., "1," "2," and "5." If a digit's syllable final is similar to that of the non-syllable initial character followed immediately, it is difficult to segment the non-syllable initial character accurately and segmentation errors tend to occur, such as the confusability between "5" and "55." Substitution errors mainly occur among "6," "9," and "yiao" ("yiao" is the variation of "1"), or between "2" and "8," because of the similarity of their syllable finals. Insertion and deletion errors are high related with the accuracy of segmentation. Hence, mandarin digit string is a good platform for verifying the all-path decoding algorithm.

The data corpus used in this experiment was the digit string database built by Institute of Automation, CAS [8]. The database was collected from 55 male speakers ( 80 utterances per speaker). The speech of the first 40 speakers (ordered by the name of speakers) were taken as the training set and the data from the remaining 15 speakers as the test set in digit string experiments.

The baseline system[5] was based on the whole-word model and each SSM was sequentially composed of 40 regions and each region was modeled by 12 Gaussian mixtures. Acoustic features were 12 dimensions MFCC plus 1 dimension normalized energy and their 1 st and 2 nd order derivatives. The comparison of mandarin digit string recognition results of two systems, the all-path decoding based system and the best path decoding based system, was listed in table1. "S.Corr," "Sub," "Del," "Ins" and "Err" were the string correct, substitution, deletion insertion and word error rate respectively. Compared with the baseline, 
Table 1. Mandarin digit string recognition results achieved by the all-path decoding based system and best path based system.

\begin{tabular}{|c|c|c|c|c|c|}
\hline Decoder & S.Corr\% & Sub\% & Del\% & Ins\% & Err\% \\
\hline Best Path & 95.00 & 1.02 & 0.27 & 0.35 & 1.64 \\
\hline All Paths & 95.67 & 0.91 & 0.23 & 0.27 & 1.41 \\
\hline
\end{tabular}

Table 2. Recognition results in Test-863 achieved by the all-path decoding based system and best path based system.

\begin{tabular}{|c|c|c|c|c|}
\hline Decoder & Sub\% & Del\% & Ins\% & CER\% \\
\hline Best Path & 12.9 & 0.1 & 0.0 & 13.0 \\
\hline All-Path & 12.4 & 0.1 & 0.1 & 12.6 \\
\hline
\end{tabular}

the all-path method reduced $14.0 \%$ relative word error rate and the total error made by insertion and deletion was obviously decreased by $19.4 \%$. It is useful to consider information from all paths instead of the best path in tasks with heavy insertion and deletion errors.

\subsection{Mandarin LVCSR}

The all-path decoding algorithm had also been run in a mandarin LVCSR system to test the algorithm performance on common tasks. The data corpus applied in LVCSR experiments was provided by Chinese National Hi-Tech Project 863 for Mandarin LVCSR system development [9]. 83 male speakers' data were employed for training (48373 sentences, 55.6 hours)and 6 male speakers' for test (240 sentences, 17.1 minutes). The acoustic feature adopted the same configures used in section 4.1 .

The baseline system was a context-dependent triphone SSM system [4]. The search paths were organized by the lexical tree and began/ended with the silence model. Each segment model was sequentially composed of 15 regions and each region was modeled by 12 Gaussian mixtures. Region models were tied by phone based decision trees. Triphone based duration models were used to improve the system accuracy.

Table 2 showed the recognition results on Test- 863 by the best path decoding and all-path decoding. The "CER" here meant the character error rate. In Test-863, the character error rate reduced $3 \%$, which was not so effective as we expected. The insertion and deletion error were also not alleviated too. It might be caused by two factors. First, the weight of sub-optimal path scores in the all-path score was limited. In Equ.(13), the gain of the fusing score by one sub-optimal path was at most $(\beta \cdot \ln 2)$, if the sub-optimal path was with an equal score as the optimal path. In practice, the optimal path score was multiple times of the sub-optimal path scores when they were transferred to probabilities. Hence, the gain by combining these sub-optimal paths was limited; the other factor was that the main error in Test- 863 was substitution error, which might 
Table 3. The difference between the results achieved by the best path decoding and all-path decoding.

\begin{tabular}{|c|c|c|c|c|}
\hline Mode & Sub\% & Del\% & Ins\% & Err\% \\
\hline B-A & 3.2 & 0.0 & 0.1 & 3.3 \\
\hline S-A $^{*}$ & 10.4 & 0.1 & 0.1 & 10.6 \\
\hline
\end{tabular}

come from the confusion between models, or be due to the wrong segmentation. Since the insertion and deletion error rates were low in Test-863, the main error owed to the confusion between different models. The current all-path decoding algorithm had done little to reduce such errors.

We had also compared the difference of recognition results obtained by the best path decoding and all-path decoding, as table 3 showed. The recognition hypotheses from one system was taken as the reference for alignment, and the recognition result from the other system was compared with the reference by string comparison algorithm[10]. The higher the character error rate was, the more different between two results was, and vice versa. "B-A" showed the result by taking the "best-path" hypothesis as the reference for alignment with the "all-path" hypothesis. The character error rate was $3.3 \%$, whereas the character error rate difference of two decoding results was only $0.4 \%$, compared with the true transcription file. Hence, the different places between two recognition results should be with low confidence. In order to prove this assertion, we replaced the characters, which were marked as substitution error when the string comparison was done between the two results, with the "Don't Care" symbols[10]. The "Don't Care" symbol can match any one word in the template. Row "S-A*" gave the result obtained by using the true transcription as reference for alignment with the modified "all-path" hypothesis. Ignoring these unstable characters, the substitution error reduced $16.1 \%$ and the character error rate reduced $15.1 \%$. Though two decoding results were similar, the differences between two results were informative for low confidential characters. The result achieved by one decoding algorithm was a good complement for the other decoding result.

\section{Conclusions}

An all-path decoding algorithm, which uses the information of all possible paths or segmentations instead of the best path to recognize the speech utterance, is proposed in this paper. Compared with the conventional best path decoding algorithm, the all-path decoding algorithm has following characteristics,

- The weakness of the dividable assumption is partially amended in decoding by fusing information from all possible paths;

- The decoding result is directly based on Equ.(2), and the best path is not necessary.

- The results obtained by the all-path decoding and the best path decoding are informative and the difference identifies the characters with low confidence. 
In above experiments, the all-path decoding algorithm achieved at least a comparable result with that of the best path based algorithm. In tasks dominated by substitution errors, the experiment showed that the approximation by Equ.(3) performed as well as the original scheme, while the all-path decoding algorithm achieved a better performance than the conventional best-path based algorithm in tasks with heavy insertion and deletion errors. Considering the allpath decoding algorithm will not add obvious computation load, it is desirable for SM based decoding.

In this paper, the same acoustic model was taken in both the all-path based decoding and the best path based decoding. The acoustic model was built as the conventional way, that was, it took the dividable assumption during modeling. Such modeling method might introduce mismatch between the model and the decoding algorithm. Our following work will concentrate on building an uniform framework for speech recognition without taking the dividable assumption, that is, the corresponding segments of neighboring acoustic events may be overlapped both in modeling and decoding. We believe it would be useful for a more accurate representation of human speech.

\section{Thanks}

This work is supported in part by the China National Nature Science Foundation (No. 60172055, No. 60121302), the Beijing Nature Science Foundation (No.4042025) and the National Fundamental Research Program (973) of China (2004CB318105). The authors would like to thank the anonymous reviewers for their useful comments.

\section{References}

1. Gao, S., Lee, T., Wong, Y.W., Xu, B.: Acoustic modeling for chinese speech recognition: A comparative study of mandarin and cantonese. In: Proceedings of International Conference on Acoustics, Speech, and Signal Processing. Volume 3., Istanbul (2000) 1261-1264

2. Ostendorf, M., Roukos, S.: A stochastic segment model for phoneme based continuous speech recognition. IEEE Transactions on Acoustics, Speech and Signal Processing 37(12) (1989) 1857-1869

3. Ostendorf, M., Digalakis, V., Kimball, O.: From HMM's to segment models: A unified view of stochastic modeling for speech recognition. IEEE Transactions on Speech Audio Processing 4(5) (1996) 360-378

4. Tang, Y., Liu, W.J., Zhang, H., Xu, B., Ding, G.H.: One-pass coarse-to-fine segmental speech decoding algorithm. In: IEEE Proceedings of International Conference on Acoustics, Speech, and Signal Processing, Toulouse, France (2006) 441-444

5. Tang, Y., Liu, W.J., Zhang, Y.Y., Xu, B.: A framework for fast segment model by avoidance of redundant computation on segment. In: International Symposium on Chinese Spoken Language Processing, Hong Kong (2004) 117-121

6. Tang, Y., Zhang, H., Liu, W.J., Xu, B.: Coloring the speech utterance to accelerate the SM based LVCSR decoding. In: IEEE Proceedings of International 
Conference on Natural Language Processing and Knowledge Engineering, Wuhan, China (2005) 121-126

7. Rabiner, L., Wilpon, J., Soong, F.: High performance connected digit recognition using hidden markov models. IEEE Transactions on Acoustics, Speech, and Signal Processing 37(8) (1989) 1214-1225

8. Deng, Y.G., Huang, T.Y., Xu, B.: Towards high performance continuous mandarin digit string recognition. In: Proceedings of the International Conference on Spoken Language Processing, Beijing, China (2000) 642-645

9. Gao, S., Xu, B., Zhang, H., Zhao, B., Li, C.R., Huang, T.Y.: Update of progress of sinohear: Advanced mandarin lvcsr system at NLPR. In: Proceedings of the International Conference on Spoken Language Processing, Beijing, China (2000) 798-801

10. Duda, R., Hart, P., Stork, D.: Pattern Recognition. Second edn. John Wiley \& Sons, Inc. (2001) 